19,11

\title{
Влияние дейтерирования на фазовые переходы в $\left(\mathrm{NH}_{4}\right)_{3} \mathrm{VOF}_{5}$
}

\author{
(C) Е.В. Богданов ${ }^{1,2}$, В.С. Бондарев ${ }^{1,3}$, М.В. Горев ${ }^{1,3}$, М.С. Молокеев ${ }^{1,3}$, И.Н. Флёров ${ }^{1,3}$ \\ ${ }^{1}$ Институт фоизики им. Л.В. Киренского СО РАН - обособленное подразделение ФИЦ КНЦ СО РАН, \\ Красноярск, Россия \\ ${ }^{2}$ Институт инженерных систем и энергетики, Красноярский государственный аграрный университет, \\ Красноярск, Россия \\ ${ }^{3}$ Институт инженерной физики и радиоэлектроники, Сибирский федеральный университет, \\ Красноярск, Россия \\ E-mail: evbogdanov@iph.krasn.ru
}

Поступила в Редакцию 11 ноября 2021 г.

В окончательной редакции 11 ноября 2021 г.

Принята к публикации 13 ноября 2021 г.

Выращен $\left(\mathrm{ND}_{4}\right)_{3} \mathrm{VOF}_{5}$ с высокой степенью дейтерирования $(D \approx 92 \%)$. Выполнены структурные и теплофизические исследования, определены параметры фазовых переходов. Установлено, что дейтерирование аммонийного катиона в $\left(\mathrm{NH}_{4}\right)_{3} \mathrm{VOF}_{5}$ привело к изменению химического давления, которое сопровождается увеличением объема элементарной ячейки и ростом температур фазовых переходов. Определены барические коэффициенты $d T_{i} / d p$ и построена фазовая $T-p$ диаграмма $\left(\mathrm{ND}_{4}\right)_{3} \mathrm{VOF}_{5}$. Обнаружено уменьшение температурной устойчивости исходной кубической фазы $F m \overline{3} m$ в $\left(\mathrm{ND}_{4}\right)_{3} \mathrm{VOF}_{5}$, а также выклинивание промежуточной моноклинной фазы при более низком давлении по сравнению с $\left(\mathrm{NH}_{4}\right)_{3} \mathrm{VOF}_{5}$ Существенное уменьшение в результате дейтерирования изменения энтропии при фазовых переходах свидетельствует в пользу уменьшения ангармонизма колебаний аммонийных тетраэдров.

Ключевые слова: оксифториды, фазовые переходы, теплоемкость, тепловое расширение, давление.

DOI: 10.21883/FTT.2022.03.52101.237

\section{1. Введение}

Кристаллическая структура ряда комплексных оксифторидов состоит из полярных анионов $\left[M \mathrm{O}_{x} \mathrm{~F}_{6-x}\right][1]$, наличие которых может привести к появлению макроскопического дипольного момента в объемном кристалле и созданию новых полярных материалов. Близость размеров и электроотрицательности кислородных и фторных лигандов приводит к разупорядочению фторкислородных октаэдров и макроскопический дипольный момент в структуре оксифторидов в целом реализуется достаточно редко [2]. Один из путей получения упорядоченных структур фторкислородных материалов заключается в создании окружения шести-координированных полиэдров, приводящего к упорядочению лигандов [3-5]. Методология создания упорядоченных оксифторидов состоит в понимании характера разупорядочения трехмерной кристаллической структуры и прогнозировании возможной ориентации локальных полярных моментов [6].

Кристаллы семейства оксифторида ванадия, достаточно многообразны, благодаря способности ванадия менять валентное состояние и формировать различные полярные/неполярные фторкислородные анионы $\left[\mathrm{VO}_{x} \mathrm{~F}_{6-x}\right]$ [7-11]. Наличие двух типов связи центрального атома в октаэдре $(\mathrm{V}-\mathrm{O}$ и $\mathrm{V}-\mathrm{F})$ могло бы приводить к возможности возникновения дипольного момента. Комплексные исследования показали, что кристаллы $\left(\mathrm{NH}_{4}\right)_{3} \mathrm{VOF}_{5}$ и $\left(\mathrm{NH}_{4}\right)_{3} \mathrm{VO}_{2} \mathrm{~F}_{4}$ имеют два независимых фторкислородных аниона в структуре и при комнатной температуре характеризуются ромбической симметрией (пр.гр. Immm) [12,13]. Выше комнатной температуры кристаллы претерпевают переходы в высокосимметричные динамически разупорядоченные фазы (пр.гр. $F m \overline{3} m)$ с шестью и двенадцатью пространственными ориентациями фторкислородного октаэдра соответственно для $\left(\mathrm{NH}_{4}\right)_{3} \mathrm{VOF}_{5}$ и $\left(\mathrm{NH}_{4}\right)_{3} \mathrm{VO}_{2} \mathrm{~F}_{4}$. Аммонийные тетраэдры разупорядочены и/или частично упорядочены в исходной кубической и первой искаженной ромбической фазах. При охлаждении кристаллы претерпевают последовательность фазовых превращений, сопровождающихся изменением симметрии: $\left(\mathrm{NH}_{4}\right)_{3} \mathrm{VO}_{2} \mathrm{~F}_{4}-F m \overline{3} m \leftrightarrow \operatorname{Immm}(I 222) \leftrightarrow$ ромбичес$\kappa a я \leftrightarrow P 112 / m \leftrightarrow P \overline{1} ; \quad\left(\mathrm{NH}_{4}\right)_{3} \mathrm{VOF}_{5}-F m \overline{3} m \leftrightarrow I m m m \leftrightarrow$ $\leftrightarrow ? \leftrightarrow P \overline{1} \quad[14,15]$. Данные колебательной спектроскопии подтверждают динамический характер разупорядочения структуры, а полное замораживание ориентационного движения аммонийных групп наблюдается в кристаллах $\left(\mathrm{NH}_{4}\right)_{3} \mathrm{VOF}_{5}$ и $\left(\mathrm{NH}_{4}\right)_{3} \mathrm{VO}_{2} \mathrm{~F}_{4}$ в районе $T \sim 35 \mathrm{~K}$ [16]. Изменение валентности центрального атома $\left(\mathrm{V}^{4+} \rightarrow \mathrm{V}^{5+}\right)$, сопровождающееся ростом его ионного радиуса $\left(C_{N}=6-R_{\mathrm{V}^{4+}}=0.58 \mathrm{~A} ; R_{\mathrm{V}^{5+}}=0.54 \mathrm{~A}\right)$, приводит к уменьшению химического давления в $\left(\mathrm{NH}_{4}\right)_{3} \mathrm{VOF}_{5}$ и увеличению $(\sim 1.5 \%)$ объема элементарной ячейки по сравнению с $\left(\mathrm{NH}_{4}\right)_{3} \mathrm{VO}_{2} \mathrm{~F}_{4}$, а также к ряду особенностей в поведении физических свойств. В частности происходит значительное изменение, во-первых, температур фазовых переходов, во-вторых, чувствительности исходной и искаженных фаз к гидростатическому давлению, в-третьих, суммарной энтропии фазовых пре- 
вращений $[14,15]$. Последнее обстоятельство согласуется с моделью разупорядочения структурных элементов в исходной кубической фазе $F m \overline{3} m[13]$. При этом природа структурных искажений остается сегнетоэластической (несегнетоэлектрической).

Возможность изменения степени разупорядочения фторкислородных анионов показана на примере замещения катиона $A, A^{\prime}=\mathrm{NH}_{4}, \mathrm{ND}_{4}, \mathrm{~K}, \mathrm{Cs}, \mathrm{Rb}$ в межоктаэдрической полости соединений $A A^{\prime} M \mathrm{O}_{2} \mathrm{~F}_{4}[17,18]$. Дейтерирование аммонийной группы в $\left(\mathrm{NH}_{4}\right)_{3} \mathrm{VO}_{2} \mathrm{~F}_{4}$ привело к уменьшению химического давления, при этом объем элементарной ячейки увеличился $(\sim 1.5 \%)$ в $\left(\mathrm{ND}_{4}\right)_{3} \mathrm{VO}_{2} \mathrm{~F}_{4}$ по сравнению с $\left(\mathrm{NH}_{4}\right)_{3} \mathrm{VO}_{2} \mathrm{~F}_{4}$ [19]. $\mathrm{B}$ результате дейтерирования вторая ромбическая фаза в $\left(\mathrm{ND}_{4}\right)_{3} \mathrm{VO}_{2} \mathrm{~F}_{4}$ выклинивается и вместо последовательности фазовых переходов Immm $\leftrightarrow$ ромбическая $б а з а \leftrightarrow P 112 / m$ имеет место прямой переход Immm $\leftrightarrow P 112 / m$. [14]. Дейтерирование также привело к уменьшению индивидуальных энтропий фазовых переходов $F m \overline{3} m \leftrightarrow$ Immm и Immm $\leftrightarrow P 112 / m$. Величина полного изменения энтропии $\Sigma \Delta S_{i} \approx R \cdot \ln 6$ в $\left(\mathrm{ND}_{4}\right)_{3} \mathrm{VO}_{2} \mathrm{~F}_{4}$, связанная с искажением структуры при понижении симметрии кристаллической решетки от кубической до триклинной, оказалась значительно меньше, чем в протонированном соединении. Уменьшение полной энтропии $\Sigma \Delta S_{i}$ в результате дейтерирования свидетельствует о значительной роли степени ангармонизма колебаний аммонийных тетраэдров.

В настоящей работе выполнены исследования влияния дейтерирования на термодинамические свойства оксопентафторида ванадия $\left(\mathrm{NH}_{4}\right)_{3} \mathrm{VOF}_{5}$ в области фазовых переходов, происходящих в результате разупорядочения/упорядочения элементов структуры.

\section{2. Экспериментальные методы и результаты исследований}

Для получения дейтерированного соединения исходный кристалл $\left(\mathrm{NH}_{4}\right)_{3} \mathrm{VOF}_{5}$ растворялся в тяжелой воде $(\sim 99.9 \% \mathrm{D})$. Далее раствор помещался в эксикатор c $\mathrm{P}_{2} \mathrm{O}_{5}$ и выдерживался там до полного поглощения воды. Процесс перекристаллизации в тяжелой воде повторялся несколько раз для достижения максимальной степени дейтерирования. Процентное содержание дейтерия $\mathrm{D} \approx 92 \%$ было определено путем сравнения интенсивностей линий поглощения ЯМР ${ }^{1} \mathrm{H}$ протонного и дейтерированного соединений [20].

Порошковая рентгенограмма $\left(\mathrm{ND}_{4}\right)_{3} \mathrm{VOF}_{5}$ получена при комнатной температуре на дифрактометре D8 ADVANCE фирмы Bruker, с использованием линейного детектора VANTEC и $\mathrm{Cu}-\mathrm{K} \alpha$ излучение (рис. $1, a$ ). Шаг детектора был равен $0.016^{\circ}$, экспозиция в каждой точке $0.6 \mathrm{~s}$. Основные рефлексы на рентгенограмме были проиндицированы в ромбической структуре (пр.гр. Immm), с параметрами близкими для $\left(\mathrm{NH}_{4}\right)_{3} \mathrm{VO}_{2} \mathrm{~F}_{4}$ [12]. Поэтому структура этого оксифторида была использована в качестве исходной модели

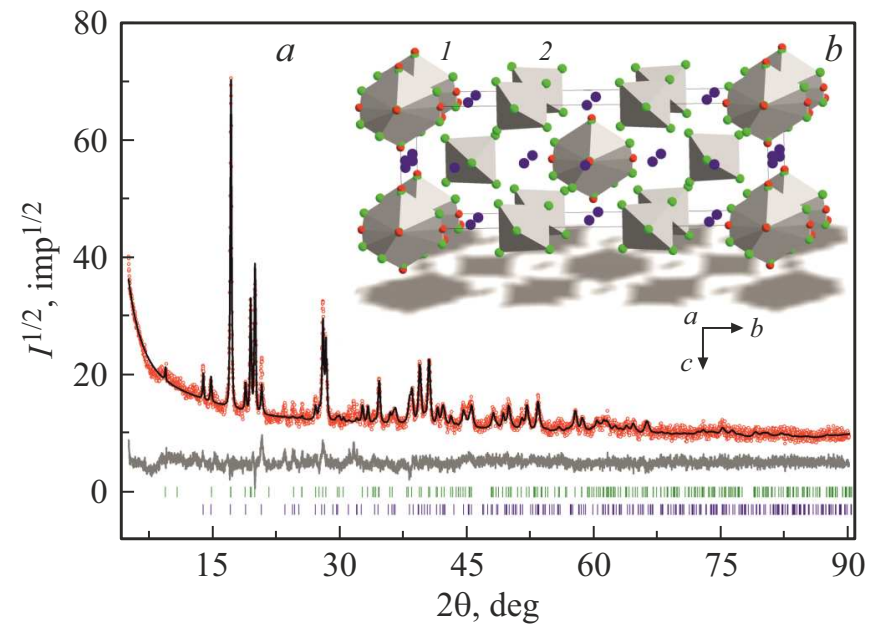

Рис. 1. Результаты уточнения структуры $\left(\mathrm{ND}_{4}\right)_{3} \mathrm{OF}_{5}$ методом Ритвельда $(a)$. Кристаллическая структура $(b)\left(1-\mathrm{VOF}_{5}\right.$ разупорядочен по многим позициям, $2-\mathrm{VOF}_{5}$ разупорядочен по двум позициям).

для уточнения. Единственное изменение было связано с коррекцией заселенностей позиций атомов фтора и кислорода для того чтобы конечная формула соответствовала $\left(\mathrm{ND}_{4}\right)_{3} \mathrm{VOF}_{5}$. В итоге, изначально полностью упорядоченный полиэдр $\mathrm{VO}_{2} \mathrm{~F}_{4}$ пришлось разупорядочить по 2 позициям, чтобы получить полиэдр $\mathrm{VOF}_{5}$, так как через $\mathrm{VO}_{2} \mathrm{~F}_{4}$ проходила плоскость симметрии $\mathrm{m}$, не позволявшая упорядочить группу $\mathrm{VOF}_{5}$ (рис. $1, b$ ). На рентгенограмме также обнаружено несколько слабых пиков, не принадлежащих фазе $\left(\mathrm{ND}_{4}\right)_{3} \mathrm{VOF}_{5}$. Часть из них были ассоциированы с фазой $\left(\mathrm{NH}_{4}\right)_{2} \mathrm{VOF}_{4}$, которой оказалось $\sim 13 \%$ по весу. Уточнение методом Ритвельда выполнено в программе TOPAS 4.2 [21] и дало низкие значения $R$ факторов (табл. 1 , рис. $1, a$ ).

В результате исследования температурной зависимости теплового расширения получена информация о наличии и температурах фазовых превращений в $\left(\mathrm{ND}_{4}\right)_{3} \mathrm{VOF}_{5}$. Измерения проводились на индукционном дилатометре NETZSCH DIL-402C в температурном диапазоне $120-420 \mathrm{~K}$ в динамическом режиме со скоростью нагрева $\sim 3 \mathrm{~K} / \mathrm{min}$, в потоке газообразного гелия

Таблица 1. Основные параметры уточнения структуры кристалла $\left(\mathrm{ND}_{4}\right)_{3} \mathrm{VOF}_{5}$

\begin{tabular}{c|c}
\hline Пр.гр. & Immm \\
\hline$a, \AA$ & $9.1553(10)$ \\
$b, \AA$ & $18.929(4)$ \\
$c, \AA$ & $6.3072(13)$ \\
$V, \AA^{3}$ & $1093.1(3)$ \\
$2 \theta$ интервал, & $5-90$ \\
$R_{\text {wр }}, \%$ & 10.17 \\
$R_{\mathrm{p}}, \%$ & 7.83 \\
$R_{\mathrm{B}}, \%$ & 4.85 \\
$\chi^{2}$ & 2.09
\end{tabular}


при расходе $\sim 50 \mathrm{ml} / \mathrm{min}$. Для калибровки прибора и учета теплового расширения измерительной системы использовались эталоны из плавленого кварца [22]. Исследуемые образцы были приготовлены из поликристаллического $\left(\mathrm{ND}_{4}\right)_{3} \mathrm{VOF}_{5}$ в виде таблеток диаметром $\sim 4 \mathrm{~mm}$ и высотой $\sim 4-6 \mathrm{~mm}$ путем прессования при давлении $\sim 2 \mathrm{GPa}$.

Температурное поведение деформации и коэффициента объемного расширения $\left(\mathrm{ND}_{4}\right)_{3} \mathrm{VOF}_{5}$ (рис. 2) качественно согласуются с зависимостями $\Delta V / V_{0}(T)$ и $\beta(T)$, полученными ранее для $\left(\mathrm{NH}_{4}\right)_{3} \mathrm{VOF}_{5}$ [15]. Дополнительных аномалий, вызванных наличием небольшого количества примеси, обнаружено не было. Ряд отличий связан с величинами изменения деформации и температурами фазовых переходов. Аномальное поведение деформации в $\left(\mathrm{ND}_{4}\right)_{3} \mathrm{VOF}_{5}$ при $T_{1}=355 \pm 1 \mathrm{~K}$, связанное с переходом первого рода, сопровождается

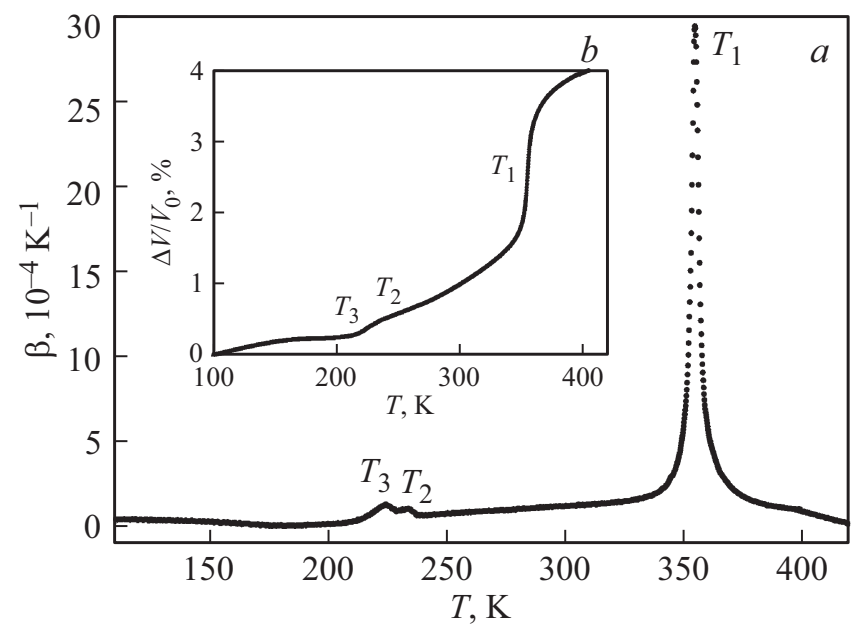

Pис. 2. Температурные зависимости коэффициента объемного расширения $(a)$ и деформации $(b)\left(\mathrm{ND}_{4}\right)_{3} \mathrm{VOF}_{5}$.

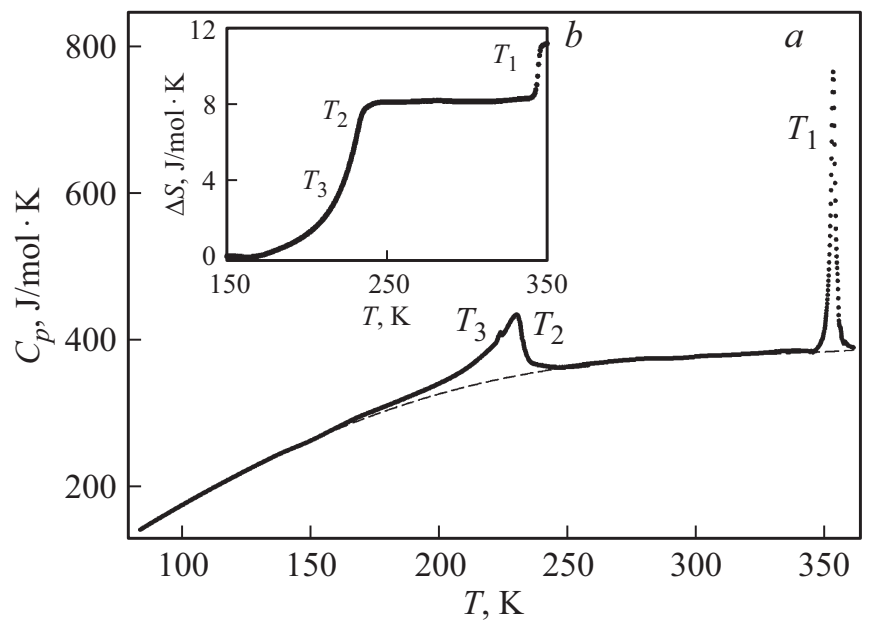

Рис. 3. Температурные зависимости теплоемкости (a) и энтропии фазовых переходов $(b)$ в $\left(\mathrm{ND}_{4}\right)_{3} \mathrm{VOF}_{5}$ в широком интервале температур. Штриховая линия - решеточная теплоемкость.
Таблица 2. Термодинамические параметры фазовых переходов в оксифторидах $\left(\mathrm{NH}_{4}\right)_{3} \mathrm{VOF}_{5}$ и $\left(\mathrm{ND}_{4}\right)_{3} \mathrm{VOF}_{5}$

\begin{tabular}{c|c|c}
\hline Параметр & $\left(\mathrm{NH}_{4}\right)_{3} \mathrm{VOF}_{5}[15]$ & $\left(\mathrm{ND}_{4}\right)_{3} \mathrm{VOF}_{5}$ \\
\hline$T_{1}, \mathrm{~K}$ & $348.1 \pm 0.5$ & $353.2 \pm 0.5$ \\
$\Delta S_{1}, \mathrm{~J} / \mathrm{mol} \cdot \mathrm{K}$ & $5.4 \pm 0.5$ & $3.0 \pm 0.3$ \\
$\left(d T_{1} / d p\right)_{\text {calc }}, \mathrm{K} / \mathrm{GPa}$ & 115 & 93 \\
$T_{2}, \mathrm{~K}$ & $229.1 \pm 0.2$ & $230.2 \pm 0.2$ \\
$\left(A_{T}^{2} / B\right)_{T 2}, \mathrm{~J} / \mathrm{mol} \cdot \mathrm{K}^{2}$ & -0.6 & -0.5 \\
$\left(A_{T}^{3} / C\right)_{T 2}, \mathrm{~J}^{2} / \mathrm{mol}^{2} \cdot \mathrm{K}^{3}$ & 4.6 & 6.3 \\
$T_{2}-T_{C 2}, \mathrm{~K}$ & 3.2 & 6.3 \\
$N_{2}$ & -0.13 & -0.19 \\
$\Delta S_{2}, \mathrm{~J} / \mathrm{mol} \cdot \mathrm{K}$ & $7.6 \pm 0.7$ & $7.9 \pm 0.7$ \\
$\left(d T_{2} / d p\right)_{\text {exp }}, \mathrm{K} / \mathrm{GPa}$ & $23 \pm 2$ & - \\
$\left(d T_{2} / d p\right)_{\text {calc }}, \mathrm{K} / \mathrm{GPa}$ & 26 & 13 \\
$T_{3}, \mathrm{~K}$ & $218 \pm 1$ & $224 \pm 1$ \\
$\left(A_{T}^{2} / B\right)_{T 3}, \mathrm{~J} / \mathrm{mol} \cdot \mathrm{K}^{2}$ & - & -0.29 \\
$\left(A_{T}{ }^{3} / C\right)_{T 3}, \mathrm{~J}^{2} / \mathrm{mol}{ }^{2} \cdot \mathrm{K}^{3}$ & - & 2.1 \\
$T_{3}-T_{C 3}, \mathrm{~K}$ & - & 6.1 \\
$\mathrm{~N}_{3}$ & - & -0.19 \\
$\Delta S_{3}, \mathrm{~J} / \mathrm{mol} \cdot \mathrm{K}$ & $0.4 \pm 0.06$ & $0.2 \pm 0.05$ \\
$\left(d T_{3} / d p\right)_{\text {exp }}, \mathrm{K} / \mathrm{GPa}$ & $92 \pm 4$ & - \\
$\left(d T_{3} / d p\right)_{\text {calc }}, \mathrm{K} / \mathrm{GPa}$ & 120 & 88
\end{tabular}

значительным скачком $\delta\left(V / V_{0}\right)\left(T_{1}\right)=2 \cdot 10^{-2}$ (рис. $\left.2, b\right)$. В низкотемпературной области наблюдается последовательность из двух слабовыраженных аномалий, которые более наглядно проявляются на температурной зависимости коэффициента объемного теплового расширения: $\Delta \beta\left(T_{2}\right)=0.25 \cdot 10^{-4} 1 / \mathrm{K}$ и $\Delta \beta\left(T_{3}\right)=0.65 \cdot 10^{-4} 1 / \mathrm{K}$ (рис. 2, $a$ ).

Исследования температурной зависимости теплоемкости $C_{p}(T)\left(\mathrm{ND}_{4}\right)_{3} \mathrm{VOF}_{5}$ методом адиабатической калориметрии позволили уточнить температуры фазовых переходов и определить их энергетические и энтропийные характеристики. Поликристаллический образец с общей массой $\sim 0.3 \mathrm{~g}$ герметично упаковывался в инертной атмосфере гелия в фурнитуру с нагревателем. Измерения теплоемкости системы проводились в режимах непрерывных $(d T / d t=0.15 \mathrm{~K} / \mathrm{min})$ и дискретных $(\Delta T=2.5-3.0 \mathrm{~K})$ нагревов. Теплоемкость фурнитуры измерялась в отдельном эксперименте.

Температурное поведение молярной изобарной теплоемкости $\left(\mathrm{NH}_{4}\right)_{3} \mathrm{VOF}_{5}$ (рис. $\left.3, a\right)$ качественно согласуется с $C_{p}(T)$ зависимостью, полученной для протонированного соединения [15]. Сравнение уточненных температур фазовых переходов, реализующихся в $\left(\mathrm{NH}_{4}\right)_{3} \mathrm{VOF}_{5}$ и $\left(\mathrm{ND}_{4}\right)_{3} \mathrm{VOF}_{5}$, показывает, что дейтерирование привело к их росту (табл. 2 ).

\section{3. Обсуждение результатов}

Высокая степень дейтерирования оксопентафторида ванадия $\left(\mathrm{NH}_{4}\right)_{3} \mathrm{VOF}_{5}(\sim 92 \% \mathrm{D})$ не привела к изменению симметрии реализующейся при комнатной температуре ромбической фазы (пр.гр. Iтmm). При этом 
объем элементарной ячейки $\left(\mathrm{ND}_{4}\right)_{3} \mathrm{VOF}_{5}$ по сравнению с протонированным ванадатом $\left(\mathrm{NH}_{4}\right)_{3} \mathrm{VOF}_{5}$ увеличился всего на $\sim 0.2 \%$. Полученное значение существенно отличается от величины изменения объема элементарной ячейки, которым сопровождалось дейтерирование диоксотетрафторида ванадия $\left(\mathrm{NH}_{4}\right)_{3} \mathrm{VO}_{2} \mathrm{~F}_{4}(\sim 1.5 \%)$ [19]. Небольшая величина изменения объема при дейтерировании аммонийного катиона наблюдалась в системе оксифторидов $\left(\mathrm{NH}_{4}\right)_{2} \mathrm{MO}_{2} \mathrm{~F}_{4}(M=\mathrm{Mo}, \mathrm{W})$ с изолированными октаэдрами $[20,23]$. Оценка изменения химического давления $\Delta p \approx \Delta T /(d T / d p)$ в $\left(\mathrm{ND}_{4}\right)_{3} \mathrm{VOF}_{5}$, выполнена с использованием фазовой $T-p$ диаграммы исходного кристалла $\left(\mathrm{NH}_{4}\right)_{3} \mathrm{VOF}_{5}$ и изменений температур фазовых превращений в результате замещения $\mathrm{D} \rightarrow \mathrm{H}$. Полученная величина $\Delta p \approx 0.1 \mathrm{GPa}$ оказалась достаточно близка к значению $(\Delta p \approx-0.08 \mathrm{GPa})$, полученному ранее при исследовании влияния дейтерирования $\left(\mathrm{NH}_{4}\right)_{3} \mathrm{VO}_{2} \mathrm{~F}_{4}$. Таким образом, аммонийные оксифториды ванадия, структура которых состоит из связанных октаэдров $\left[\mathrm{VO}_{x} \mathrm{~F}_{6-x}\right]$, характеризуются значительно большей чувствительностью к изменению химического давления, возникающему в результате замещения $\mathrm{D} \rightarrow \mathrm{H}$, чем аммонийные оксифториды $\left(\mathrm{NH}_{4}\right)_{2} \mathrm{WO}_{2} \mathrm{~F}_{4}$ и $\left(\mathrm{NH}_{4}\right)_{2} \mathrm{MoO}_{2} \mathrm{~F}_{4}$ с изолированными октаэдрами $(\Delta p \approx 0.02 \mathrm{GPa})[20,23]$.

Для определения энергетических и энтропийных характеристик фазовых переходов в $\left(\mathrm{ND}_{4}\right)_{3} \mathrm{VOF}_{5}$ выполнено разделение теплоемкости на регулярный $C_{\mathrm{reg}}$ и аномальный $\Delta C_{p}(T)$, связанный с последовательностью переходов, вклады. Для этого участки температурной зависимости теплоемкости вне области существования аномалий рассматривались как соответствующие $C_{\text {reg }}$ и аппроксимировались комбинацией функций Дебая и Эйнштейна. Интерполяция функции $C_{\mathrm{reg}}(T)$ на области аномального поведения $C_{p}(T)$ позволила определить избыточную теплоемкость (рис. 3, $a$ и 4, $a$ ). Величины изменения энтропии $\Delta S_{i}=\int\left(\Delta C_{p} / T\right) d T$, полученные в результате интегрирования температурной зависимости $\Delta C_{p}(T)$, представлены в табл. 2.

Дейтерирование $\left(\mathrm{NH}_{4}\right)_{3} \mathrm{VOF}_{5}$ привело к существенному уменьшению энтропии высокотемпературного фазового превращения $\Delta S_{1}$ (табл. 2), что косвенно подтверждает его связь с процессами упорядочения как фтор-кислородных октаэдров, так и аммонийных тетраэдров [15]. Аномальное поведение теплофизических свойств в низкотемпературной области, характерное для фазовых переходов второго рода, не изменилось. Это обстоятельство позволило выполнить анализ температурной зависимости избыточной теплоемкости $\Delta C_{p}(T)$ в рамках термодинамической теории Ландау [24] (рис. 4, $a$ ) и определить величины изменения энтропии $\Delta S_{2}$ и $\Delta S_{3}$, а также ряд других параметров фазовых переходов при $T_{2}$ и $T_{3}$. Квадрат обратной избыточной теплоемкости вблизи низкотемпературных фазовых переходов в $\left(\mathrm{ND}_{4}\right)_{3} \mathrm{VOF}_{5}$ достаточно хорошо описывается линейной функцией температуры (рис. $4, b):\left(\Delta C_{p} / T\right)^{-2}=\left[2 \cdot\left(B^{2}\right.\right.$ $\left.\left.-3 \cdot A^{\prime} C\right)^{0.5} / A_{T}^{2}\right]^{2}+12 \cdot C\left(T_{0}-T\right) / A_{T}{ }^{3}$, где величины $A=A_{T} \cdot\left(T_{0}-T_{C}\right)+A_{T} \cdot\left(T-T_{0}\right)=A^{\prime}+A_{T} \cdot\left(T-T_{0}\right), B$

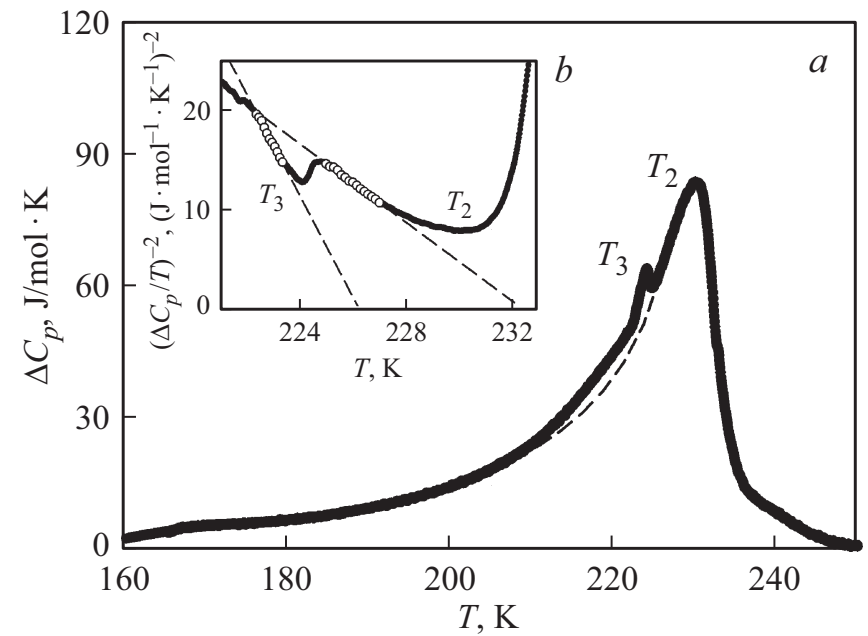

Рис. 4. Температурные зависимости избыточной теплоемкости $(a)$ и квадрата обратной ее величины $(b)$ в окрестности $T_{2}$ и $T_{3}$.

и $C$ являются коэффициентами термодинамического потенциала: $\Delta \Phi(p, T, \eta)=A \cdot \eta^{2}+B \cdot \eta^{4}+C \cdot{ }^{6}(\eta-$ параметр перехода, $T_{C}$ - температура Кюри, $T_{0}$ - температура фазового перехода).

Оказалось, что энтропии низкотемпературных фазовых превращений в результате замещения $\mathrm{D} \rightarrow \mathrm{H}$ практически не изменились (табл. 2), что, в свою очередь, подтверждает преимущественный вклад фтор-кислородных октаэдров в механизм фазовых переходов типа упорядочения и смещения соответственно при $T_{2}$ и $T_{3}$.

Сравнение феноменологических параметров ванадиевых оксифторидов показало (табл. 2), что в результате дейтерирования низкотемпературные превращения в $\left(\mathrm{ND}_{4}\right)_{3} \mathrm{VOF}_{5}$ оказались значительно дальше от трикритической точки $\left(T_{i}-T_{C i}=0\right.$ и $N=0$, где $N= \pm\left[B^{2} /\left(3 \cdot A_{T} C T_{0}\right)\right]^{-0.5}-$ степень близости перехода к трикритической точке), чем соответствующие фазовые переходы в $\left(\mathrm{NH}_{4}\right)_{3} \mathrm{VOF}_{5}$ [15].

Совместный анализ экспериментальных калориметрических и дилатометрических данных позволил оценить величины барических коэффициентов $d T_{i} / d p$ в $\left(\mathrm{ND}_{4}\right)_{3} \mathrm{VOF}_{5}$ (табл. 2). Величина $d T_{1} / d p$ для высокотемпературного превращения рассчитана из уравнения Клапейрона-Клаузиуса для переходов первого рода, а коэффициенты $d T_{2} / d p$ и $d T_{3} / d p$ для фазовых переходов второго рода определены в рамках соотношения Эренфеста $\left(\Delta C_{p}=\Delta \beta \cdot T_{i} /\left(d T_{i} / d p\right)_{p}=0\right)[25]$.

Аналогичные оценки барических коэффициентов, выполненные ранее для $\left(\mathrm{NH}_{4}\right)_{3} \mathrm{VOF}_{5}[15]$, показали хорошее согласие между рассчитанными величинами $d T_{i} / d p$ и полученными путем прямых измерений.

На основе данных о температурах фазовых переходов и вычисленных барических коэффициентах построена фазовая $T-p$ диаграмма $\left(\mathrm{ND}_{4}\right)_{3} \mathrm{VOF}_{5}$ (рис. 5), которая качественно соответствует диаграмме протонированного кристалла $\left(\mathrm{NH}_{4}\right)_{3} \mathrm{VOF}_{5}$. Таким образом, можно сделать 


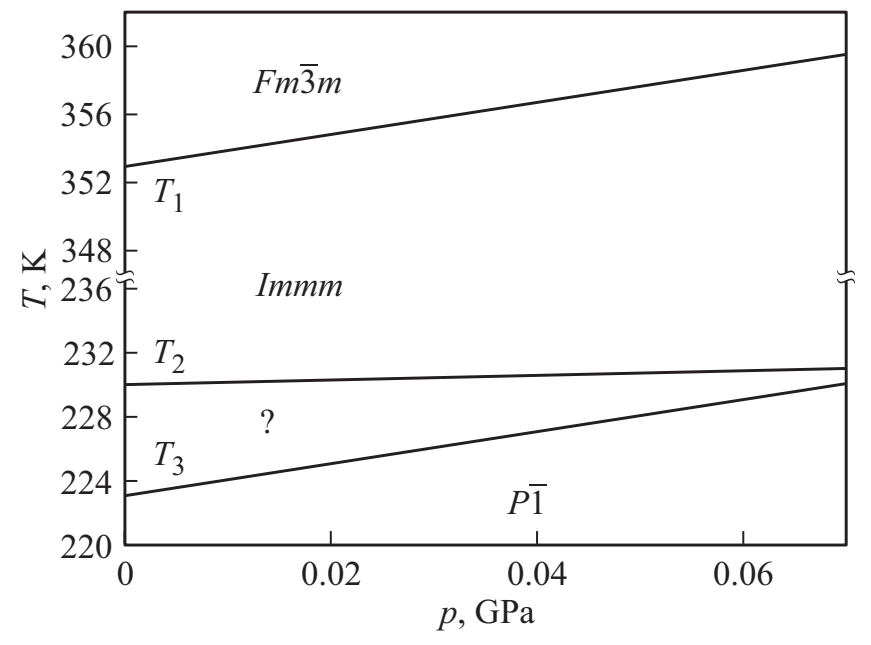

Рис. 5. Фазовая диаграмма температура-давление кристалла $\left(\mathrm{ND}_{4}\right)_{3} \mathrm{VOF}_{5}$, построенная на основе расчета барических коэффициентов $\left(d T_{i} / d p\right)_{\text {calc }}$.

вывод, о том, что дейтерирование аммонийного катиона не привело к изменению симметрии фаз и последовательности сегнетоэластических фазовых переходов $F m \overline{3} m \leftrightarrow$ Immm $\leftrightarrow$ моноклинная $\leftrightarrow P \overline{1}(P 1)$.

Дейтерирование $\left(\mathrm{NH}_{4}\right)_{3} \mathrm{VOF}_{5}$ приводит к увеличению температуры $T_{1}$ высокотемпературного фазового перехода в $\left(\mathrm{ND}_{4}\right)_{3} \mathrm{VOF}_{5}$, которое сопровождается уменьшением барического коэффициента $d T_{1} / d p$. В результате на фазовой $T-p$ диаграмме $\left(\mathrm{ND}_{4}\right)_{3} \mathrm{VOF}_{5}$ область существования исходной кубической фазы (пр. гр. $F m \overline{3} m$ ) сужается, а ромбическая фаза (пр. гр. Immm) становится энергетически выгодной в более широком интервале температур. Замещении $\mathrm{D} \rightarrow \mathrm{H}$ оказывает влияние как на температуры $T_{2}$ и $T_{3}$, так и на величины барических коэффициентов $d T_{2} / d p$ и $d T_{3} / d p$. В результате, на фазовой $T-p$ диаграмме $\left(\mathrm{ND}_{4}\right)_{3} \mathrm{VOF}_{5}$ происходит сужение области стабильности промежуточной моноклинной фазы и последующее выклинивание при увеличении внешнего давления. Тройная точка в $\left(\mathrm{ND}_{4}\right)_{3} \mathrm{VOF}_{5}$ реализуется при меньшем значении избыточного давления $p_{t r} \approx 0.08 \mathrm{GPa}$ $\left(p_{t r} \approx 0.1 \mathrm{GPa}\right.$ в случае $\left.\left(\mathrm{NH}_{4}\right)_{3} \mathrm{VOF}_{5}[15]\right)$. Изменение области стабильности высокотемпературной кубической фазы и смещение тройной точки в $\left(\mathrm{ND}_{4}\right)_{3} \mathrm{VOF}_{5}$ в области низких давлений согласуется с поведением фазовых переходов в $\left(\mathrm{NH}_{4}\right)_{3} \mathrm{VOF}_{5}$ при увеличении внешнего гидростатического давления.

Комплексные исследования дейтерированых кристаллов $\left(\mathrm{ND}_{4}\right)_{3} \mathrm{VOF}_{5}$ и $\left(\mathrm{ND}_{4}\right)_{3} \mathrm{VO}_{2} \mathrm{~F}_{4}$ [15] показали, что замещение $\mathrm{D} \rightarrow \mathrm{H}$ приводит не только к изменению температур фазовых переходов, но и к существенному изменению структурных, энергетических и барических характеристик фазовых переходов, происходящих в результате изменения химического давления. С другой стороны, химическое давление, связанное с дейтерированием аммонийного катиона, не приводит к изменению природы и механизмов фазовых превращений.
Сегнетоэластические фазовые переходы, реализующиеся в оксифторидах ванадия, сопровождаются значительными величинами изменения энтропии $\Sigma \Delta S_{i}$ и барических коэффициентов $d T_{i} / d p$. Поэтому соединения $\left(\mathrm{NH}_{4}\right)_{3} \mathrm{VOF}_{5}$ и $\left(\mathrm{NH}_{4}\right)_{3} \mathrm{VO}_{2} \mathrm{~F}_{4}$ могут представлять интерес с точки зрения исследования их барокалорической эффективности.

\section{4. Заключение}

Выращены кристаллы дейтерированного оксопентафторида ванадия (IV), $\left(\mathrm{ND}_{4}\right)_{3} \mathrm{VOF}_{5}$, и выполнены исследования теплофизических свойств в широком интервале температур.

1. Установлено, что замещения $\mathrm{D} \rightarrow \mathrm{H}$ не повлияло на последовательность сегнетоэластических фазовых переходов в $\left(\mathrm{ND}_{4}\right)_{3} \mathrm{VOF}_{5}$.

2. Дейтерирование привело к изменению химического давления, которое сопровождается увеличением объема элементарной ячейки и ростом температур фазовых переходов в $\left(\mathrm{ND}_{4}\right)_{3} \mathrm{VOF}_{5}$.

3. Выполнена оценка барических коэффициентов $d T_{i} / d p$ и построена предполагаемая фазовая $T-p$ диаграмма.

4. Величина избыточного химического давления в $\left(\mathrm{ND}_{4}\right)_{3} \mathrm{VOF}_{5}$ определена на основе анализа фазовой $T-p$ диаграммы $\left(\mathrm{NH}_{4}\right)_{3} \mathrm{VOF}_{5}$ и оказалась близка к величине изменения давления, возникающего при дейтерировании $\left(\mathrm{NH}_{4}\right)_{3} \mathrm{VOF}_{5}$.

5. Установлено уменьшение температурной устойчивости исходной кубической фазы в $\left(\mathrm{ND}_{4}\right)_{3} \mathrm{VOF}_{5}$, а также выклинивание промежуточной моноклинной фазы при более низком давлении по сравнению с $\left(\mathrm{NH}_{4}\right)_{3} \mathrm{VOF}_{5}$.

6. Дейтерирование $\left(\mathrm{NH}_{4}\right)_{3} \mathrm{VOF}_{5}$ приводит к уменьшению величины $\Delta S_{1}$, что косвенно подтверждает связь фазового перехода при $T_{1}$ с процессами упорядочения фтор-кислородного октаэдра и аммонийных тетраэдров.

7. Показано, что низкотемпературные превращения в $\left(\mathrm{ND}_{4}\right)_{3} \mathrm{VOF}_{5}$ в результате дейтерирования характеризуются меньшей степенью близости к трикритической точки, чем соответствующие переходы в $\left(\mathrm{NH}_{4}\right)_{3} \mathrm{VOF}_{5}$.

8. Уменьшение величины полного изменения энтропии $\Sigma \Delta S_{i} \approx R \cdot \ln 4 \quad$ в $\left(\mathrm{ND}_{4}\right)_{3} \mathrm{VOF}_{5} \quad$ в $\Sigma \Delta S_{i} \approx R \cdot \ln 6$ в $\left(\mathrm{NH}_{4}\right)_{3} \mathrm{VOF}_{5}$ свидетельствует об участии аммонийного катиона в процессах упорядочения структуры, а также о возможности уменьшения степени ангармонизма колебаний аммонийных тетраэдров в результате дейтерирования.

\section{Благодарности}

Рентгенографические и дилатометрические данные получены с использованием оборудования Красноярского регионального центра коллективного пользования ФИЦ КНЦ СО РАН.

\section{Конфликт интересов}

Авторы заявляют, что у них нет конфликта интересов. 


\section{Список литературы}

[1] G. Pausewang, K. Dehnicke. Z. Anorg. Allg. Chem. 369, 265 (1969).

[2] N.F. Stephens, M. Buck, P. Lightfoot. J. Mater. Chem. 15, 4298 (2005).

[3] F.H. Aidoudi, C. Black, K.S. Athukorala Arachchige, A.M.Z. Slawin, R.E. Morris, P. Lightfoot. Dalton Trans. 43, 568 (2014).

[4] R. Gautier, M.D. Donakowski, K.R. Poeppelmeier. J. Solid State Chem. 195, 132 (2012).

[5] G.A. Senchyk, V.O. Bukhan'ko, A.B. Lysenko, H. Krautscheid, B. Rusanov, A.N. Chernega, M. Karbowiak, K.V. Domasevitch. Inorg. Chem. 51, 8025 (2012).

[6] R.A.F. Pinlac, M.R. Marvel, J.J.-M. Lesage, K.R. Poeppelmeier. Mater. Res. Soc. Symp. Proc. 1148, PP01-04 (2009).

[7] Р.Л. Давидович, Л.Г. Харламова, Л.В. Самарец. Координационная химия 3, 850 (1977).

[8] B.R. Wani, U.R.K. Rao, K.S. Venkateswarlu, A.S. Gokhale. Thermochim. Acta 58, 87 (1982).

[9] K. Kobayashi, T. Matsuo, H. Suga, S. Khairoun, A. Tressaud. Solid State Commun. 53, 719 (1985).

[10] P. Bukovec, N. Bukovec, A. Demšar. J. Therm. Anal. 36, 1751 (1990).

[11] B. Dojer, M. Kristl, Z. Jagličić, M. Drofenik, A. Meden. Acta Chim. Slov. 55, 834 (2008).

[11] S.J. Patwe, S.N. Achary, K.G. Girija, C.G.S. Pillai, A.K. Tyagi. J. Mater. Res. 25, 1251 (2010).

[12] M. Leimkühler, R.J. Mattes. Solid State Chem. 65, 260 (1986).

[13] A.A. Udovenko, E.I. Pogoreltsev, Y.V. Marchenko, N.M. Laptash Acta Cryst. B 73, 1 (2017).

[14] V.D. Fokina, M.V. Gorev, A.G. Kocharova, E.I. Pogoreltsev, I.N. Flerov. Solid State Sci. 11, 836 (2009).

[15] Е.В. Богданов, Е.И. Погорельцев, А.В. Карташев, М.В. Горев, М.С. Молокеев, С.В. Мельникова, И.Н. Флеров, H.M. Лапташ. ФТТ В 62, 7, 1123 (2020).

[16] Yu.V. Gerasimova, A.S. Oreshonkov, N.M. Laptash, A.N. Vtyurin, A.S. Krylov, N.P. Shestakov, A.A. Ershov, A.G. Kocharova. Spectrochim. Acta A 176, 106 (2017).

[17] Е.В. Богданов, А.Д. Васильев, И.Н. Флёров, Н.М. Лапташ. ФTT 53, 2, 284 (2011).

[18] E.V. Bogdanov, E.I. Pogoreltsev, M.V. Gorev, I.N. Flerov. Inorg. Chem. 56, 11, 6706 (2017).

[19] Е.В. Богданов, Е.И. Погорельцев, М.В. Горев, М.С. Молокеев, И.Н. Флеров. ФТТ 61, 2, 330 (2019).

[20] E.V. Bogdanov, S.V. Mel'nikova, E.I. Pogoreltsev, M.S. Molokeev, I.N. Flerov. Solid State Sci. 61, 155 (2016).

[21] Bruker AXS TOPAS V4: General profile and structure analysis software for powder diffraction data. User's Manual. Bruker AXS, Karlsruhe, Germany. (2008).

[22] М.В. Горев, Е.В. Богданов, И.Н. Флёров, А.Г. Кочарова, Н.M. Лапташ. ФТТ 52, 1, 156 (2010)

[23] И.Н. Флёров, В.Д. Фокина, М.В. Горев, Е.В. Богданов, М.С. Молокеев, А.Ф. Бовина, А.Г. Кочарова. ФТТ 49, 6, 1093 (2007).

[24] К.С. Александров, И.Н. Флёров. ФТТ 21, 327 (1979)

[25] Н. Парсонидж, Л. Стейвли. Беспорядок в кристаллах. Мир, М. (1982) 436 с.

Редактор Ю.Э. Китаев 